\title{
Small Heat Shock Protein
}

National Cancer Institute

\section{Source}

National Cancer Institute. Small Heat Shock Protein. NCI Thesaurus. Code C136829.

A family of chaperone proteins with subunit molecular masses of 12-43 kDa. The members are characterized by the presence of a highly conserved stretch of 80-100 amino acids in their C-terminal domains called the alpha-crystallin domain (ACD). Class I small heat shock proteins (sHsps) are widely expressed and play a role in cell survival. Class II sHsps are expressed in tissue-specific patterns and may be involved in the differentiation, development and specific functionality for the tissues they are expressed in. 